\title{
Constitutive $\beta$ cell expression of IL-12 does not perturb self- tolerance but intensifies established autoimmune diabetes
}

\author{
Andreas Holz, Kelly Brett, and Michael B.A. Oldstone \\ Viral-Immunobiology Laboratory, Division of Virology, Department of Neuropharmacology, The Scripps Research Institute, \\ La Jolla, California, USA \\ Address correspondence to: Michael Oldstone, Division of Virology, Department of Neuropharmacology, IMM6, \\ The Scripps Research Institute, 10550 North Torrey Pines Road, La Jolla, California 92037, USA. \\ Phone: (858) 784-8054; Fax: (858) 784-9981; E-mail: mbaobo@scripps.edu.
}

Andreas Holz's present address is: Department of Neuroimmunology, Max-Planck-Institute of Neurobiology, Martinsried, Germany.

Kelly Brett's present address is: Graduate Program in Immunology, Washington University School of Medicine, St. Louis, Missouri, USA.

Received for publication August 6, 2001, and accepted in revised form October 15, 2001.

To analyze the function of the Th1-promoting cytokine IL-12 in vivo, we generated transgenic ( $\mathrm{tg})$ mice (RIP-IL12 mice) whose pancreatic $\beta$ cells constitutively express bioactive IL-12 or one of its components, p35 or p40. In contrast to non-tg littermates or single-tg RIP-p35 and RIP-p40 mice, RIP-IL12 mice developed a marked pancreatic infiltration of lymphocytes and macrophages, mainly around islets. Expression of bioactive IL-12 primarily upregulated transcript levels of IFN-inducible protein-10 (IP-10), RANTES, IFN- $\gamma$, and TNF- $\alpha$ in the pancreas. Despite the substantial recruitment of mononuclear cells, no biochemical or clinical disease was evident in the exocrine or endocrine pancreas. Coexpression of lymphocytic choriomeningitis virus (LCMV) proteins with IL-12 in the $\beta$ cells failed to spontaneously activate or expand antigen-specific anti-self/viral $\mathrm{T}$ cells in uninfected tg animals. However, when RIP-IL12 $\times$ RIP-LCMV tg mice were infected with LCMV, antigen-specific anti-self/viral T cells were induced, which led to an acceleration in the kinetics and severity of insulin-dependent diabetes mellitus (IDDM). Thus, the ectopic expression of IL-12 does not spontaneously break tolerance and activate antigen-specific $\mathrm{T}$ cells in the periphery, but it does worsen ongoing autoimmune disease.

J. Clin. Invest. 108:1749-1758 (2001). DOI:10.1172/JCI200113915.

\section{Introduction}

After encountering a pathogen, the cytokine IL-12 acts as both an inducer and a regulator of innate and adoptive immune responses (1). Antigen-presenting cells such as macrophages and dendritic cells produce IL-12, which in turn induces $\mathrm{T}$ lymphocytes and natural killer cells to generate high levels of IFN- $\gamma(2,3)$. IL-12 causes naive Th lymphocytes to differentiate into Th1 cells (4-6). Therefore, IL-12 is believed to promote T cell-mediated autoimmune diseases, such as insulindependent diabetes mellitus (IDDM) and multiple sclerosis (reviewed in refs. 7, 8). Antibodies and gene ablation that neutralize the activity of IL-12 in vivo protect against autoimmunity (9-11), and the administration of bioactive IL-12 worsens autoimmune disease $(12,13)$.

Earlier, we prepared transgenic $(\mathrm{tg})$ mice whose pancreatic $\beta$ cells expressed the glycoprotein (GP) or nucleoprotein (NP) of lymphocytic choriomeningitis virus (LCMV). When these $\beta$ cells simultaneously expressed IFN- $\gamma$ or B7.1 $(14,15)$, previously unresponsive antigenspecific anti-self/virus $\mathrm{T}$ cells spontaneously activated and caused IDDM. Others $(16,17)$ have also shown that local expression of IFN- $\gamma$ in $\beta$ cells of normal mice was associated with the onset of spontaneous IDDM and a break of immunologic tolerance.
Although IFN- $\gamma$ is the direct downstream mediator of IL-12 production, the consequences of local constitutive expression of IL-12 on breaking peripheral tolerance and causing autoimmune disease are unknown. Therefore, we used an insulin promoter to create tg mice in which the cytokine IL-12 is constitutively produced in $\beta$ cells of the pancreatic islets of Langerhans. Our results indicate that local IL-12 production results in a significant Th1 mononuclear cell response and inflammation in the pancreas, but is unable to break immunologic tolerance of antigen-specific $T$ cells located in the periphery, or to cause spontaneous autoimmune disease. However, once initiated, IDDM progressed significantly faster and with greater ferocity in the presence of IL-12.

\section{Methods}

Generation and screening of RIP-IL12 tg mice, RAG-1 knockout mice, RIP-GP mice, and RIP-NP mice. Murine IL-12p35 and IL-12p40 cDNAs (obtained from Genetics Institute Inc., Cambridge, Massachusetts, USA) were digested with the restriction enzymes PstI/EcoRI and ClaI/EcoRI, respectively. After treatment with Klenow fragment, each cDNA was cloned individually, under control of the insulin promoter (from Nora Sarvetnick, 
The Scripps Research Institute) (Figure 1). Correct integration of the IL-12 cDNAs was confirmed by sequence analysis. The IL-12p35 and IL-12p40 insulin promoter constructs were linearized by restriction digest and gelpurified. They were then comicroinjected into approximately $200 \mathrm{C} 57 \mathrm{BL} / 6 \times \mathrm{BALB} / \mathrm{c}\left(\mathrm{H}-2^{\mathrm{b} \times d}\right)$ fertilized oocytes, and implanted into pseudopregnant isogeneic females. Offspring were screened for the integration of transgenes into the genome by two independent PCRs: a first PCR used the primer set INS-sense 5'CTGTCTCCCAGATCACTGTCCTTCTGC and p35-antisense 5'-CTGGAACTCTGTCTGGTACATCTTCAAGTCC to identify the RIP-p35 transgene; a second PCR used the primer set p40-sense 5'-AGGCTCTGGAAAGACCCTGACC and p40-antisense $5^{\prime}$-CTCCAGGAGTCAGGGTACTCCC to detect the RIP-p40 transgene.

RIP-IL12 tg mice $\left(\mathrm{C} 57 \mathrm{BL} / 6, \mathrm{H}-2^{\mathrm{b}}\right)$ were bred to C57BL/6 (H-2 $\left.{ }^{\mathrm{b}}\right)$ RAG-1 knockout mice (18). Offspring of the F2 generation were analyzed for disruption of the RAG-1 gene by PCR. First, the primer combination RAG-sense (5'-CTGCCTCCTTGCCGTCTACC- $\left.3^{\prime}\right)$ and RAG-antisense (5'-AAATCCTGGCAATGAGGTCTGGC- $3^{\prime}$ ) determined the presence of a wild-type copy of the RAG-1 gene. Second, the primer pair neo-sense ( $5^{\prime}$ GGATTGCACGCAGGTTCTCCG-3') and neo-antisense (5'-CCGGCCACAGTCGATGAATCC- $\left.3^{\prime}\right)$ identified a disrupted RAG-1 gene. FACS analysis to show loss of T cells and B cells was used to confirm the PCR results in randomly tested blood.

RIP-IL12 mice (H-2 $\left.{ }^{\text {b }}\right)$ were backcrossed to C57BL/6 $\left(\mathrm{H}-2^{\mathrm{b}}\right)$ RIP-GP and RIP-NP mice (19) for at least four generations. Offspring were genotyped for the LCMV transgenes by PCR as described (20).

Induction of IDDM in tg mice. RIP-IL12 $\times$ RIP-LCMV mice, 6-8 weeks old, received LCMV Armstrong 5 intraperitoneally (IP) $\left(10^{5}\right.$ plaque-forming units [PFU]) (19). Blood glucose values were determined by using the Accucheck III system (Roche Molecular Biochemicals, Indianapolis, Indiana, USA) (11), daily (RIP-IL12 $\times$ RIP-GP line) or weekly (RIP-IL12 $\times$ RIP-NP line). Mice with two consecutive blood glucose readings of $300 \mathrm{mg} / \mathrm{dl}$ or higher were defined as diabetic.

Determination of amylase and lipase levels. Supernatants of centrifuged, coagulated blood samples from the retro-orbital plexus of test mice were assayed for amylase and lipase activities at Biomedical Testing Services (San Diego, California, USA).

$R$ Nase protection assays. Total RNA was isolated from the pancreata of RIP-IL12 $(n=7)$, RIP-p35 $(n=3)$, and RIP-p40 $(n=4)$ mice, and from their non-tg littermates $(n=3)$. RNase protection assays (RPAs) were performed as described (11), using the probe sets mCK1, $\mathrm{mCK} 2, \mathrm{mCK} 3 \mathrm{~b}, \mathrm{mCK} 5$, and $\mathrm{mCD} 1$ (PharMingen, La Jolla, California, USA). Twenty micrograms of total RNA was hybridized to ${ }^{32} \mathrm{P}$-labeled riboprobes of the probe sets used, digested with RNase A and RNase T1, and separated on a sequencing gel. The intensity of protected RNA fragments was quantified using a Storm 860 PhosphorImager and ImageQuant soft- ware (Molecular Dynamics, Sunnyvale, California, USA). Each transcript level was normalized to the RNA of the ubiquitous housekeeping protein L32.

Adoptive transfer. Lymphocytes were recovered from pancreata or pancreatic draining lymph nodes (PDLNs) of RIP-IL12 (H-2 $\left.{ }^{\mathrm{b}}\right)$ mice and injected IP into RAG-1-deficient littermates. After 10 days, blood glucose concentrations were determined, pancreata of recipients were analyzed by immunohistochemistry, and their spleen cells were tested by FACS or immunohistochemistry to confirm RAG-1 deficiency.

Cytokine ELISA. Pancreatic islets from RIP-IL12 and non-tg littermates were isolated by collagenase $P$ digestion (21). Islets were cultured in CMRL medium for 24 hours, after which the supernatants were transferred to 96-well dishes coated with anti-mouse IL-12p35/p70 monoclonal antibody (clone G297-289; PharMingen). Subsequently, bioactive IL-12p70 was detected with biotinylated anti-mouse IL-12p40/p70 (clone C17.8; PharMingen), streptavidin-horseradish peroxidase, and 2,2'-azino-bis-(3-ethylbenzthiazoline-6-sulfonic acid) (ABST) staining (11). Serial diluents of mouse recombinant IL-12 (PharMingen) served as protein concentration standards. Plates were analyzed at $405 \mathrm{~nm}$.

Cytotoxicity assay. RIP-IL12, RIP-GP, and RIP-NP tg mice, either uninfected or inoculated IP with $10^{5} \mathrm{PFU}$ LCMV seven days before analysis, were tested for the primary cytolytic activity of splenic lymphocytes (effector cells) by ${ }^{51} \mathrm{Cr}$ release assay $(11,22)$. Effector cells were incubated for 5 hours with uninfected, ${ }^{51} \mathrm{Cr}$-labeled target cells without peptide or coated with the H-2 $\mathrm{D}^{\mathrm{b}}$-restricted LCMV peptides GP aa 33-41 and NP aa 396-404. Peptide concentration was $5 \mu \mathrm{g} / \mathrm{ml}$, and the ratios of effector cells to target cells were 100:1, 50:1, 25:1, and 12.5:1. Assays were performed in triplicate with a variance of less than $10 \%$. Allogeneic $\mathrm{H}-2^{\mathrm{d}}$ target cells served as controls and did not release specific ${ }^{51} \mathrm{Cr}$. As an alternative to the use of peptide-coated target cells, LCMV at an moi of 1 was used to infect syngeneic $\mathrm{H}-2^{\mathrm{b}}$ or allogeneic $\mathrm{H}-2^{\mathrm{d}}$ target cells. Calculation of specific ${ }^{51} \mathrm{Cr}$ release was performed as described (20).

For secondary cytotoxicity (CTL) assays, effector cells were cultured with 2 units/ml recombinant IL-2, 2 $\mu \mathrm{g} / \mathrm{ml}$ GP aa 33-41 or NP aa 396-404, and irradiated syngeneic antigen-presenting cells. After 5 days, cytolytic activity was determined using ${ }^{51} \mathrm{Cr}$-labeled syngeneic and allogeneic target cells, at effector cell to target cell ratios of 5:1 and 1:1 $(11,19)$.

FACS analysis. For FACS analysis, suspensions of single cells were isolated from pancreata, PDLNs, or spleens. Cells were incubated in 10\% FBS/RPMI (Gibco BRL; Life Technologies Inc., Carlsbad, California, USA), stimulated in the presence of brefeldin A (1 $\mu \mathrm{g} / \mathrm{ml}$; Sigma-Aldrich, St. Louis, Missouri, USA), either overnight using LCMV peptides $\left(\mathrm{H}-2^{\mathrm{b}}\right.$-restricted: GP aa 33-41, NP aa 396-404; I-A $\mathrm{A}^{\mathrm{b}}$-restricted: GP aa 61-80, NP aa 309-328), or for 5 hours using $5 \mathrm{ng} / \mathrm{ml}$ PMA and $500 \mathrm{ng} / \mathrm{ml}$ ionomycin. Thereafter, cells were 
a
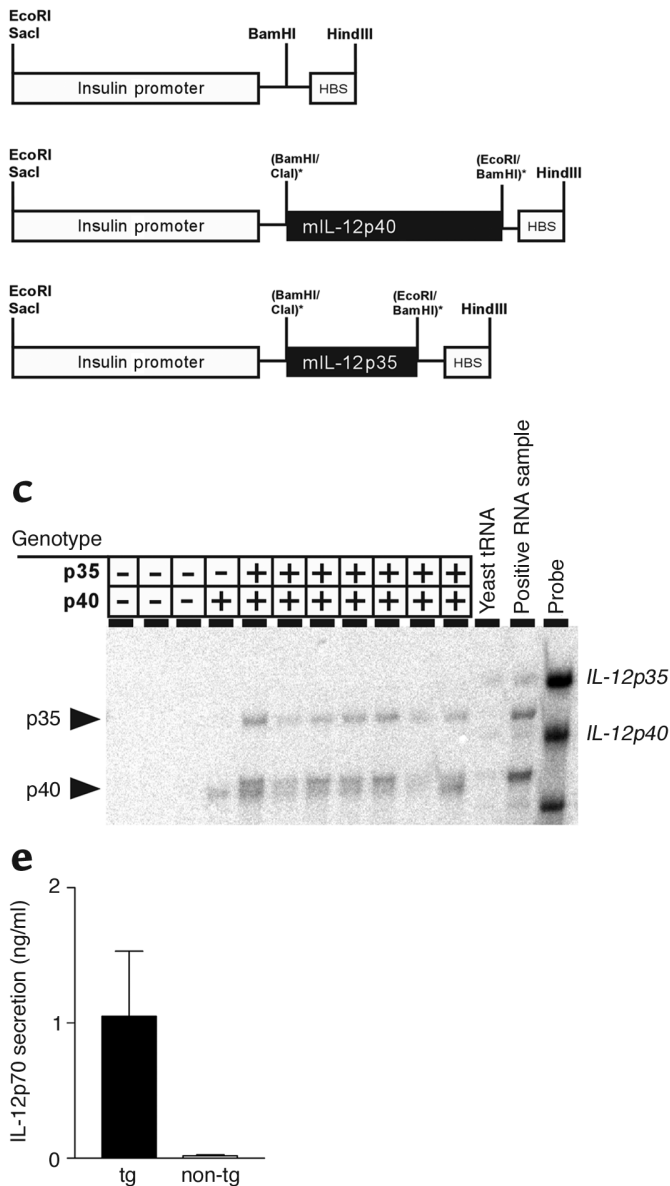

b
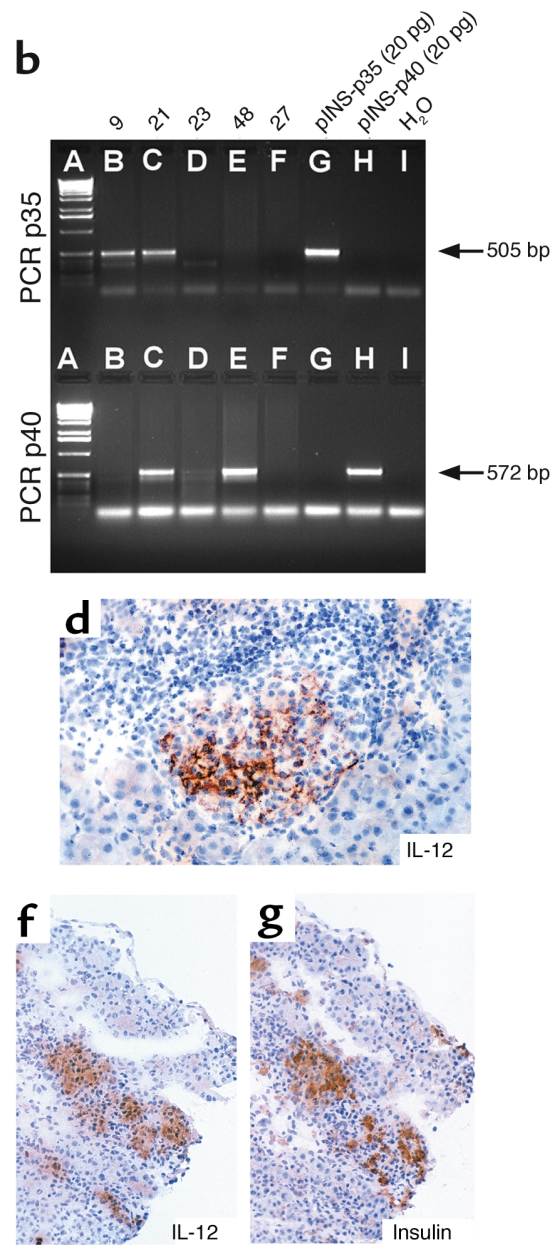

\section{Figure 1}

Generation and characterization of RIP-IL12 tg mice. (a) The murine IL-12 monomers p35 and p40 were placed behind the insulin promoter (16) using the indicated restriction sites, and comicroinjected into fertilized eggs of C57BL/ $6 \times \mathrm{BALB} / \mathrm{c}\left(\mathrm{H}-2^{\mathrm{b} \times d}\right)$ mice. (b) PCR analysis of the genomic DNA of offspring demonstrated distinct tg lines that carried either both RIP-IL12 transgenes (e.g., line 21) or the RIP-p35 or RIP-p40 transgenes alone (line 9 and line 48, respectively). Lines 23 and 27 showed no tg integration into their genomes. The RIP-p35 and RIP-p40 plasmid constructs (positive controls for PCR) demonstrated the specificity of the technique (lanes G and H). (c) RPA of non-tg $(n=3)$ or $\operatorname{tg}(n=8)$ mice revealed specific transcription of the RIP-IL12 transgenes in the pancreata of tg offspring. The arrows indicate the calculated size of the IL-12p35- and IL-12p40-protected RNA fragments. Note that RIP-p40 tg mice expressed only p40 RNA ( $n=4)$, and RIP-p35 mice $(n=3)$ had only p35 mRNA in the pancreas (see also Figure 3 ). (d) Immunohistochemical staining for IL-12 in pancreata of 4-month-old RIP-IL12 tg mice $(n=4)$ demonstrates focal IL-12 in the islets. (e) By ELISA, bioactive IL-12p70 was found to be secreted from purified islets of RIP-IL12 tg mice but not those of non-tg littermates. (f) Neonatal (1 day old) RIP-IL12 mice expressed IL-12 colocalized with insulin expressed on an adjacent section ( $\mathbf{g})$. HSB, hepatitis B terminator sequence.

stained for surface markers with either anti-mouse CD3 (145-2C11), anti-mouse CD4 (L3T4), or antimouse CD8 (Ly-2) antibodies (PharMingen) (11). Cells were then permeabilized and fixed with $4 \%$ paraformaldehyde and $0.1 \%$ saponin in PBS. Intracellularly trapped cytokines were detected with monoclonal antibodies specific for mouse IFN- $\gamma$ (XMG1.2), TNF- $\alpha$ (MPG-XT22), and IL-4 (11B11). Stained cells were analyzed using a FACSCalibur flow cytometer and CellQuest software (Becton Dickinson Immunocytometry Systems, San Jose, California, USA).

Histology and immunohistochemistry. Pancreatic tissues from RIP-IL12 and non-tg littermate mice $(n=4 ; 8-26$ weeks old) were fixed overnight in Bouin's solution, embedded in paraffin, sectioned, and stained with hematoxylin and eosin. For immunohistochemical analysis, pancreata were quick-frozen in TissueTek (Sakura, Torrance, California, USA). Tissue sections (8 $\mu \mathrm{m})$ were stained with antibodies to murine IL-12 (polyclonal goat serum; R\&D Systems Inc., Minneapolis, Minnesota, USA), insulin (guinea pig; DAKO Corp., Carpinteria, California, USA), CD4 (L3T4; PharMingen), CD8 (Ly-2 and Ly-3.2; PharMingen), F4/80 (C1:A3-1; Serotec, Raleigh, North Carolina, USA), B220 (RA3-6B2; PharMingen), NLDC 145 (Accurate Chemical and Scientific Corp., Westbury, New York, USA), MHC class I (ER-HR-52; Bachem California Inc., Torrance, California, USA), and MHC 

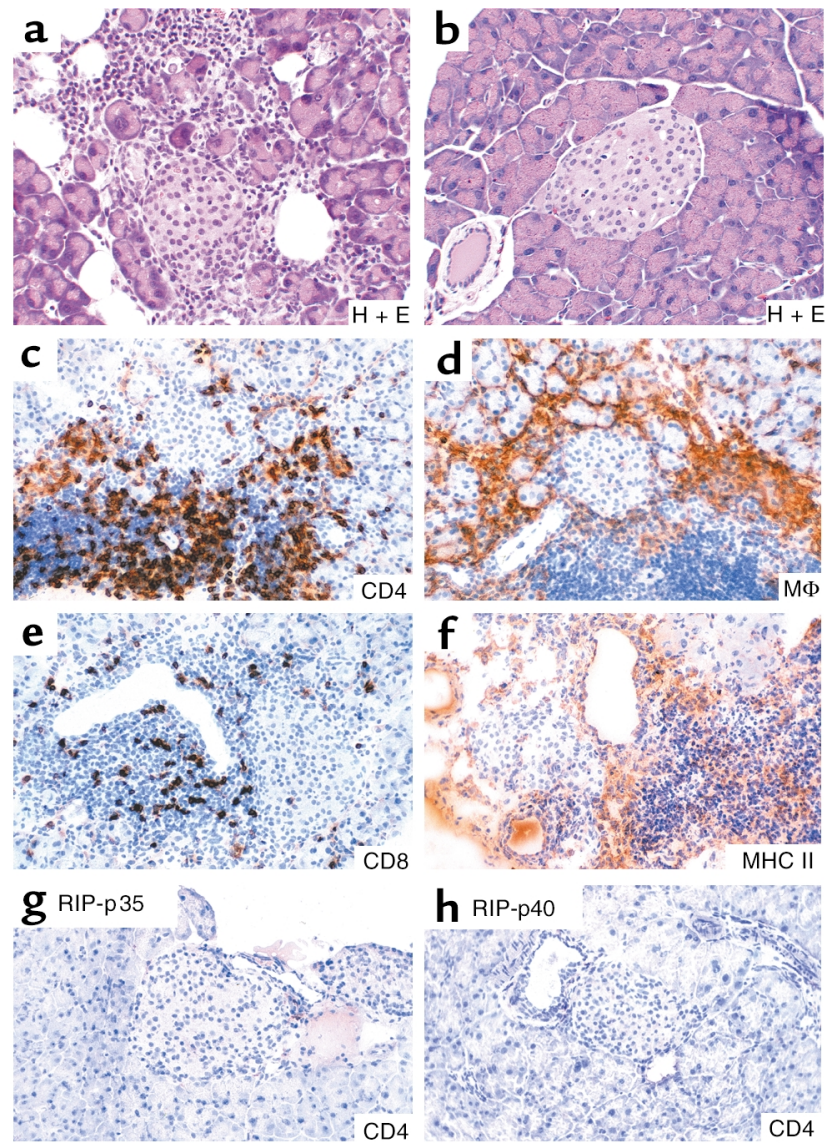

Figure 2

Spontaneous infiltration of mononuclear cells into the pancreata of RIP-IL12 tg mice. Histologic analysis showed infiltration of mononuclear cells, primarily around the islets in the pancreata of RIP-IL12 tg animals (a), but not those of non-tg littermates (b). Immunohistochemical examination revealed $\mathrm{CD}^{+}{ }^{+} \mathrm{T}$ lymphocytes (c), macrophages (d), and CD8 ${ }^{+} \mathrm{T}$ lymphocytes $(\mathbf{e})$. In general, four- to fivefold more $\mathrm{CD} 4^{+}$than $\mathrm{CD} 8^{+} T$ cells were observed in the peri-islet infiltration, with few B lymphocytes (four of four mice studied). (f) Expression of MHC class I and class II molecules was upregulated in the RIP-IL12 tg pancreas, as demonstrated for MHC class II. In contrast, the pancreata of RIP-p35 (g) and RIP-p40 (h) single-tg mice, as well as non-tg littermates, failed to show infiltration of $C D 4^{+} T$ cells ( $\mathbf{g}$ and $\mathbf{h}$, T cells) or macrophages, or enhanced expression of MHC molecules (not shown). $\mathbf{a}$ and $\mathbf{b}$ show hematoxylin and eosin staining $(\mathrm{H}+\mathrm{E})$. Counterstaining with hematoxylin is shown in $\mathbf{c}-\mathbf{h}$. Results were similar for four mice from each group.

class II (ER-TR-3; Bachem California Inc.). Sections were then stained with an appropriate species-specific, biotinylated secondary antibody (Vector Laboratories Inc., Burlingame, California, USA), a streptavidin-horseradish peroxidase complex (Vector Laboratories Inc.), and diaminobenzidine (DAB; Zymed Laboratories Inc., San Francisco, California, USA) as described (11). Slides were counterstained with hematoxylin and embedded in AquaMount (Fisher Scientific Co., Pittsburgh, Pennsylvania, USA).

Statistics. For statistical evaluation of data, Prism 3 software (GraphPad Software Inc., San Diego, California, USA) was used.

\section{Results}

$T g$ expression of IL-12 in the islets of Langerhans. We used molecular genetics to express bioactive IL-12 (2) in tg mice. Two cDNAs that encoded the complete open reading frames of the murine p 35 and p 40 monomers were individually cloned under control of the insulin promoter (Figure 1a). These constructs, termed RIP-p35 and RIP-p40, were comicroinjected into fertilized eggs and implanted into donor mice. Their offspring were analyzed for genomic integration of the transgenes. Three of 52 offspring (6\%) were positive for at least one of the two tg constructs, RIP-p35 and RIP-p40. One mouse founder line (line 21) was double-positive for both transgenes, and one line each was positive for either RIP-p35 (line 9) or RIP-p40 (line 48) (Figure 1b). These offspring were healthy, behaviorally normal, and phenotypically indistinguishable from their non-tg littermates. Mouse founder line 21 was backcrossed to C57BL/6 mice for at least six generations, with both transgenes being stably integrated and passed to progeny mice.

Analysis of pancreatic RNA showed that the RIP-p35 and RIP-p40 transgenes were specifically expressed by RIP-IL12 mice but were undetectable in non-tg littermates (Figure 1c). In single-tg offspring, only a single IL-12 RNA corresponding to the predicted genotype was detectable (Figure 1b). Immunohistochemical staining identified IL-12 protein in the pancreatic islets of RIP-IL12 tg mice (Figure 1d). No such staining was found in kidneys, livers, or brains of RIP-IL12 tg mice, or in the pancreatic islets of non-tg littermates (not shown). When single-tg RIP-p35 and RIP-p40 mice were similarly analyzed for expression of the p35 and p40 monomers, pancreatic islets again stained specifically (not shown). Hence, protein expression in all these tg lines was specific and restricted to the pancreatic islets.

As Figure 1e shows, islets purified from RIP-IL12 tg mice secreted bioactive IL-12p70, which was not detected in supernatants of cultured islets from non-tg littermates. IL-12 protein was present in the pancreata of newborn RIP-IL12 mice, and colocalized with insulin staining (Figure 1, $\mathrm{f}$ and $\mathrm{g}$ ).

Th1 cytokine-expressing mononuclear cells spontaneously infiltrate around islets and into pancreata of RIP-IL12 mice. Histologic analysis revealed mononuclear cell infiltrates surrounding islets and within exocrine areas of pancreata from RIP-IL12 tg mice. In contrast, pancreata of non-tg littermates remained free of detectable infiltration (Figure 2, $a$ and $b$ ).

As demonstrated by immunohistochemical stains, most infiltrating lymphocytes were $\mathrm{CD}^{+} \mathrm{T}$ lymphocytes (Figure $2 \mathrm{c}$ ), although $\mathrm{F} 4 / 80^{+}$macrophages (Figure $2 \mathrm{~d}$ ), $\mathrm{CD}^{+} \mathrm{T}$ cells (Figure 2e), B cells (not shown), and dendritic cells (not shown) were also present. $\mathrm{CD} 4^{+} \mathrm{T}$ cells were present at four- to fivefold greater numbers than $\mathrm{CD}^{+} \mathrm{T}$ cells were, whereas few $\mathrm{B}$ cells were observed (four of four mice studied). Infiltration was primarily around the islets of Langerhans (Figure 2,b-e). In contrast, no mononuclear cells migrated into the pancreata of either RIP-p35 or 
RIP-p40 single-tg mice (Figure 2, $\mathrm{g}$ and h). Kidney, liver, and brain tissues from RIP-IL12 $\operatorname{tg}$ mice $(n=3)$ similarly had no infiltrates (not shown).

RNA isolated from the pancreata of $\mathrm{tg}$ mice was analyzed by RPA (Figure 3), and the presence of mononuclear cells was confirmed (not shown). However, transcripts specific for mononuclear cells were below the detection limit in pancreata from non-tg littermates. Furthermore, RIP-IL12 tg mice had a greater than 20 -fold increase in the expression of multiple Th1 cytokines, i.e., IFN- $\gamma$, TNF- $\alpha$, lymphotoxin- $\beta$ (LT- $\beta$ ), and IL-1 $\beta$, compared with their non-tg littermates, single-tg RIP-p35 mice, and RIP-p40 mice (Figure 3, a and b). Less pronounced was the upregulation of IL-1 receptor $\alpha$, IFN- $\beta$, TNF- $\beta$, and TGF- $\beta$. In contrast, expression of the Th2 cytokines IL-4, IL-5, IL-9, and IL-13 was not detected in the pancreata of RIP-IL12 tg mice. However, RIP-IL12 tg mice had a 16-fold increase in the expression of IL-10 over non-tg littermates, but RIP-p35 and RIP-p40 tg mice had no rise in IL-10. In addition, transcripts of the chemokines RANTES, IFN-inducible protein 10 (IP-10), lymphotactin, $\mathrm{T}$ cell activation gene-3, and macrophage inflammatory proteins (MIPs) $1 \alpha, 1 \beta$, and 2 were markedly upregulated in RIP-IL12 tg mice, with RANTES RNA levels increased more than 60-fold over those for single-tg or control mice.

To determine the cytokine profile of lymphocytes infiltrating the pancreata of RIP-IL12 animals, flowcytometric analysis was performed. T cells isolated from the pancreata of RIP-IL12 mice displayed predominantly Th1 IFN- $\gamma$ and TNF- $\alpha$ expression in their cytoplasm; in contrast, the Th2 cytokine IL-4 was found at a low frequency (Figure 3c).

Despite T cell infiltration and upregulation of Th1 cytokines, RIP-IL12 mice show no biochemical dysfunction of blood glucose, amylase, or lipase. RIP-IL12 mice had normal endocrine and exocrine pancreas functions. As Table 1 shows, their blood glucose levels equaled those of non$\mathrm{tg}$ littermates, as did their levels of amylase and lipase. Both enzyme levels and blood glucose values were constant in young (2-4 months old) as well as older (12-16 months old) RIP-IL12 mice, and resembled those of RIP-p35, RIP-p40, and non-tg mice (Table 1).

Adoptively transferred lymphocytes from pancreata and PDLNs of RIP-IL12 tg mice migrate to pancreatic tissue of RIP-IL12 $\times R A G-1^{-/-}$mice but not RAG-1 $1^{-/-}$mice. To evaluate both their specificity and activity, lymphocytes from pancreata and the PDLNs of RIP-IL12 tg mice were adoptively transferred into RAG-1 $1^{-/}$and RIP-IL12 $\times$ RAG-1/- mice. Such lymphocytes did not home to the pancreata or islets of recipient RAG-1/- mice (Figure $4 \mathrm{a})$, and their blood glucose values remained normal $(n=4)$. In contrast, transfers into RIP-IL12 $\times$ RAG-1/mice $(n=6)$ resulted in lymphocytes migrating to and residing in peri-islet regions of the pancreas (Figure $4 \mathrm{~b}$ ). However, these lymphocytes did not cause injury to the islets, since blood glucose levels over a 1- to 2 -month observation period remained normal. These results indicated that it was unlikely that the adoptively trans- ferred lymphocytes recognized pancreatic self-antigens or that intrinsic T cells (absent in RIP-IL12 $\times$ RAG-1/mice, but present in RIP-IL12 mice) suppressed potentially autoaggressive lymphocytes. Most likely, lymphocytes from RIP-IL12 tg mice responded to the chemoattractant and cytokine molecules induced by the ectopic expression of IL-12 in the pancreas. In addition to T

a

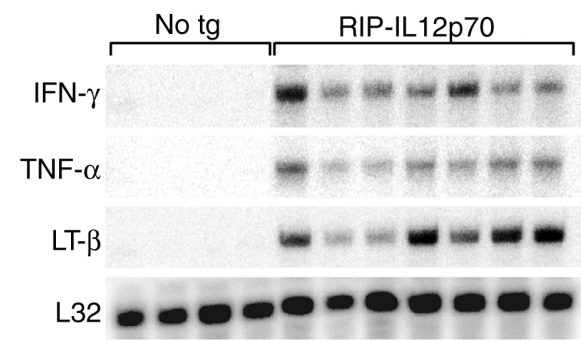

b

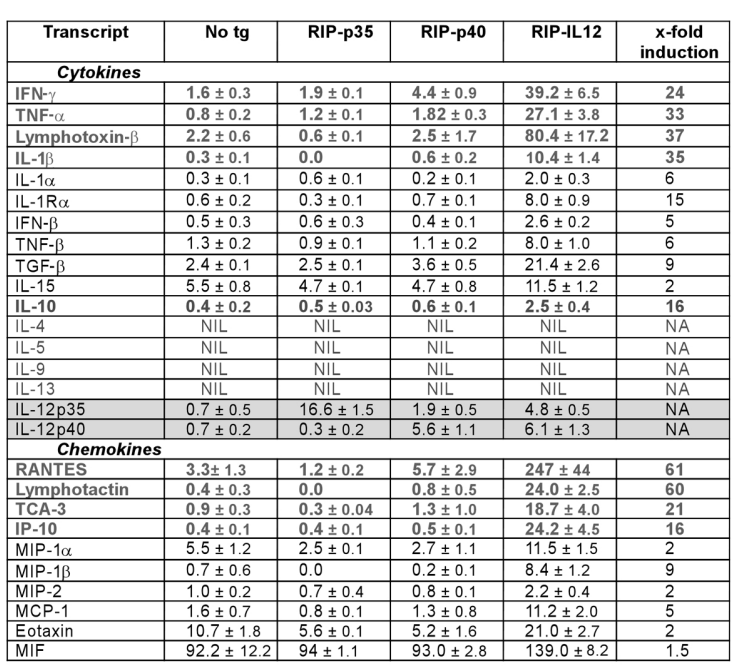

c
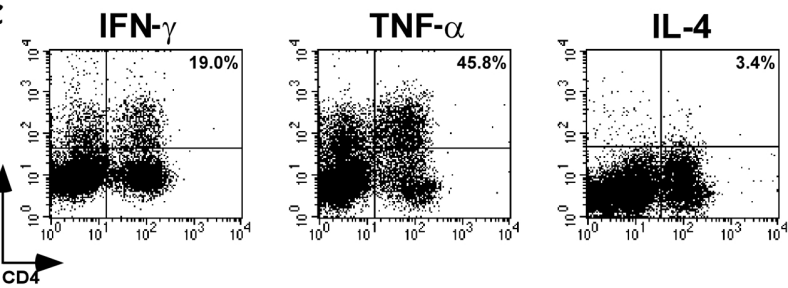

Figure 3

Expression of cytokine and chemokine transcripts in pancreata of RIP-IL12 tg mice. RNA was obtained from the pancreata of RIP-IL12 $(n=7)$, RIP-p35 $(n=3)$, and RIP-p40 $(n=4)$ tg mice and non-tg mice $(n=3)$. (a) RPA showed upregulation of IFN- $\gamma$, TNF- $\alpha$, and LT- $\beta$ in the pancreas of RIP-IL12 tg mice. All protected fragments were normalized against L32 RNA levels. (b) Shown are all transcripts analyzed, with the mean expression levels \pm SEM, and (last column) fold increase in transcription measured in RIP-IL12 tg mice over that in non-tg littermates. The Th2 cytokines IL-4, IL-5, IL-9, and IL-13 remained undetectable in all groups, whereas Th1 cytokines were markedly elevated. (c) By FACS analysis, lymphocytes isolated from the pancreata of RIP-IL12 tg mice produced primarily IFN- $\gamma$ and TNF- $\alpha$ Th1 cytokines, but not the Th2 cytokine IL- 4 after stimulation with PMA and ionomycin. The $y$ axis represents the cytokine detected, and the $x$ axis shows CD4 detection. Values display the frequency of cytokine expression among the CD4 $4^{+} \mathrm{T}$ cell population. IL-1 R $\alpha$, IL-1 receptor $\alpha$. NIL, not detectable; TCA-3, T cell activation gene-3; MIF, macrophage migration inhibitory factor. 
Table 1

RIP-IL12 tg mice show normal blood amylase, lipase, and glucose levels ${ }^{\mathrm{A}}$

\begin{tabular}{lcccccc}
\hline & \multicolumn{3}{c}{ 2-4 months of age } & \multicolumn{3}{c}{$12-16$ months of age } \\
& $\begin{array}{c}\text { Amylase } \\
(\mathrm{IU} / \mathrm{I})\end{array}$ & $\begin{array}{c}\text { Lipase } \\
(\mathrm{U} / \mathrm{l})\end{array}$ & $\begin{array}{c}\text { Blood glucose } \\
(\mathrm{mg} / \mathrm{dl})\end{array}$ & $\begin{array}{c}\text { Amylase } \\
(\mathrm{IU} / \mathrm{I})\end{array}$ & $\begin{array}{c}\text { Lipase } \\
(\mathrm{U} / \mathrm{I})\end{array}$ & $\begin{array}{c}\text { Blood glucose } \\
(\mathrm{mg} / \mathrm{dl})\end{array}$ \\
& & & & & & \\
tg & $1,494 \pm 322$ & $1,004 \pm 149$ & $164 \pm 9$ & $1,882 \pm 201$ & $1,025 \pm 86$ & $131 \pm 5$ \\
IL12p70 & $1,664 \pm 179$ & $1,151 \pm 48$ & $149 \pm 11$ & $1,431 \pm 103$ & $1,053 \pm 75$ & $136 \pm 14$ \\
p35 & $1,021 \pm 29$ & $1,137 \pm 42$ & $138 \pm 50$ & $2,286 \pm 57$ & $860 \pm 26$ & $129 \pm 11$ \\
p40 & $1,281 \pm 26$ & $1,042 \pm 97$ & $181 \pm 53$ & $1,798 \pm 114$ & $915 \pm 78$ & $153 \pm 17$
\end{tabular}

AExperimental groups were age- and sex-matched. The number of mice used in this study were: $2-4$ months old, non-tg, $n=6$; RIP-IL12, $n=13$; RIP-p35, $n=6$; RIP-p40, $n=6$. At 12-16 months old, non-tg, $n=4$; RIP-IL12, $n=12$; RIP-p35, $n=3$; RIP-p40, $n=4$. Data displayed represent the mean \pm 1 SEM.

cells, naive (untreated) RIP-IL12 $\times$ RAG-1/- $\operatorname{tg}$ mice expressed a considerable infiltration of $\mathrm{F} 4 / 80^{+}$ macrophages. By comparison, naive RAG-1/- littermates showed only a few weakly stained F4/80 cells in the pancreas (Figure 4, $\mathrm{c}$ and $\mathrm{d}$ ).

Coexpression of a self/viral-reactive antigen and IL-12 in $\beta$ cells of the pancreas does not break immunologic unresponsiveness/tolerance. RIP-LCMV GP tg mice (line 34) express the viral GP transgene only in $\beta$ cells. Potential antiself/viral $T$ cells are positively selected by the thymus and are found in the periphery as high-affinity $T$ cells in substantial numbers (15). In contrast, in a slow-onset IDDM model, characterized by RIP-LCMV NP 25.3 mice, the viral transgene is expressed in both the thymus and $\beta$ cells. Expression in the thymus results in the removal of high-affinity anti-LCMV NP T cells by negative selection, with only low-affinity anti-self/viral $\mathrm{T}$ cells being found in the periphery $(15,23)$. We then studied whether local IL-12 production in $\beta$ cells would break unresponsiveness of antigen-specific, high-affinity $T$ cells in the periphery of RIP-IL $12 \times$ RIP-GP tg mice. Such double-tg mice failed to activate antigen-specific anti-LCMV GP T cells in the absence of LCMV infection as measured by primary and secondary CTL activity (Table 2), or to develop IDDM during an observation period of over 6 months (Figure 5). RIP-IL12 $\times$ RIP-NP double-tg mice also failed to activate low-frequency anti-self/viral $\mathrm{T}$ cells in the periphery. The histologic and immunohistochemical study of naive, uninfected RIP-IL12 $\times$ RIP-LCMV double-tg mice showed a pattern of pancreatic infiltration equivalent to that observed with single-tg RIP-IL12 mice (Figure 2 and Figure 6a).

Ectopic expression of IL-12 in $\beta$ cells enhances virus-induced autoimmune diabetes. Finally, autoimmune IDDM was induced in the RIP-IL12 $\times$ RIP-LCMV double-tg mice by infection with LCMV. Viral infection elicited significant primary CTL responses in all experimental groups (Table 2 ). The incidence and kinetics of IDDM were indistinguishable in the rapid-onset RIP-GP mice with or without coexpression of IL-12 in the $\beta$ cells (Figure 5a). By contrast, LCMV infection of RIP-IL12 $\times$ RIP-NP doubletg mice accelerated the kinetics and severity of IDDM (Figure 5b). Two weeks after LCMV infection, almost $50 \%$ of RIP-IL12 $\times$ RIP-NP mice became diabetic, compared with only $6 \%$ of the RIP-NP single-tg littermates $(P<0.05)$. Eight to ten weeks after infection, more than $95 \%$ of double-tg mice had IDDM, versus $50 \%$ of singletg RIP-NP and 0\% of RIP-IL12 littermates. Thus, focal expression of IL-12 in $\beta$ cells significantly advanced the onset and progression of established IDDM.

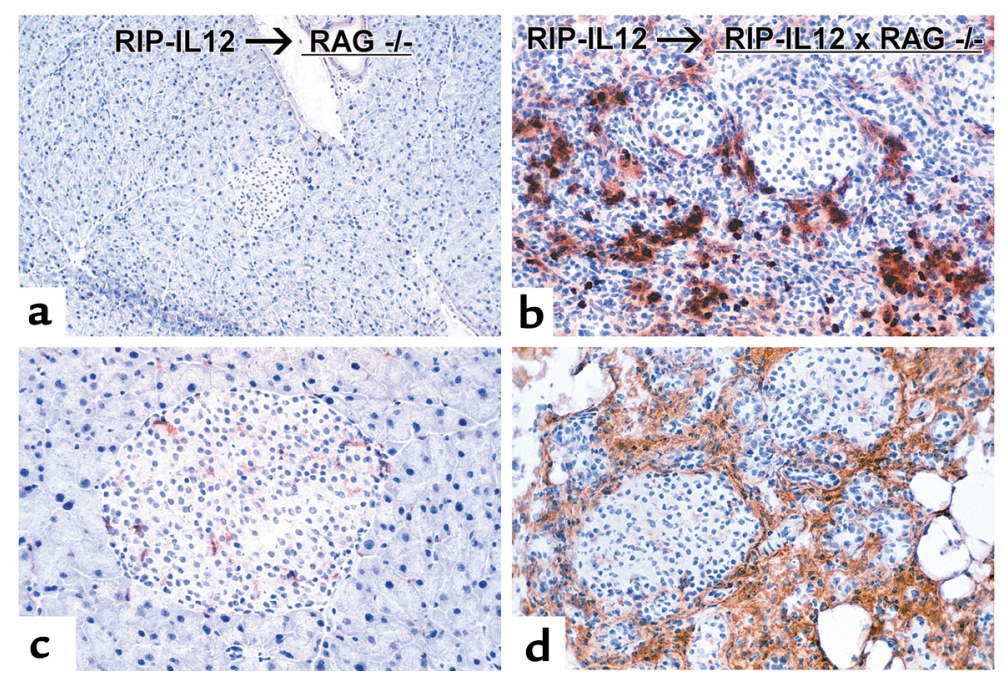

\section{Figure 4}

Adoptive transfer of lymphocytes from RIP-IL12 tg mice into RAG-1/- mice. (a and b) Lymphocytes recovered from the PDLNs and pancreata of RIP-IL12 tg mice were injected into RAG-1/- littermates. Displayed are photomicrographs of immunohistochemical staining for $\mathrm{CD} 4^{+} \mathrm{T}$ cells in the pancreas of a RAG-1\%- (a) and a RIP-IL12 $\times$ RAG-1\%- (b) recipient mouse 10 days after adoptive transfer. The transferred lymphocytes accumulated around the islets of recipient RIP-IL12 $\times$ RAG-1/-- mice, but this did not occur in a littermate that lacked ectopic expression of IL-12 by $\beta$ cells. The pancreata of RAG-1/- or RIP-IL12 $\times$ RAG-1/- mice receiving saline injections had no staining for T lymphocytes (not shown). (c and d) $\mathrm{F} 4 / 80^{+}$macrophages were detected in the pancreata of untreated RAG-1/- (c) and RIP-IL12 $\times$ RAG-1/- (d) mice. Results are representative of four mice from each group. 
Table 2

Generation of LCMV-specific CTL responses in RIP-IL12 $\times$ RIP-LCMV double-tg mice

RIP-IL12 $\times$ RIP-GPA

Experimental group RIP-GP,

day 7 after LCMV

RIP-IL12,

day 7 after LCMV

RIP-IL12 × RIP-GP,

day 7 after LCMV

RIP-IL12 × RIP-GP,

no LCMV infection

RIP-IL12 × RIP-NPD

\begin{tabular}{cccccccc} 
& \multicolumn{3}{c}{ No peptide } & \multicolumn{4}{c}{ LCMV GP aa 33-41 peptide } \\
$100: 1^{\mathrm{B}}$ & $50: 1$ & $25: 1$ & $12.5: 1$ & $100: 1$ & $50: 1$ & $25: 1$ & $12.5: 1$ \\
$8 \pm 2^{\mathrm{C}}$ & $5 \pm 1$ & $3 \pm 1$ & $2 \pm 1$ & $46 \pm 8$ & $35 \pm 9$ & $28 \pm 8$ & $20 \pm 5$ \\
& & & & & & & \\
$4 \pm 1$ & $3 \pm 1$ & $3 \pm 2$ & $2 \pm 1$ & $44 \pm 4$ & $38 \pm 5$ & $22 \pm 6$ & $17 \pm 2$ \\
$10 \pm 4$ & $7 \pm 3$ & $5 \pm 3$ & $4 \pm 2$ & $49 \pm 8$ & $33 \pm 6$ & $24 \pm 4$ & $20 \pm 3$ \\
0 & 0 & 0 & 0 & 0 & 0 & 0 & 0
\end{tabular}

LCMV NP aa 396-404 peptide

100:1 $50: 1 \quad 25: 1 \quad 12.5: 1$

$30 \pm 1 \quad 18 \pm 1 \quad 17 \pm 2 \quad 11 \pm 1$

$37 \pm 7 \quad 26 \pm 3 \quad 20 \pm 5 \quad 16 \pm 3$

$39 \pm 4 \quad 28 \pm 4 \quad 23 \pm 4 \quad 18 \pm 5$

0

0

0
Experimental group

RIP-IL12 $\times$ RIP-NP, day 7 after LCMV

RIP-IL12 $\times$ RIP-NP, no LCMV infection

RIP-IL12 $\times$ RIP-NP, no LCMV infection, 5 days in vitro culture

\begin{tabular}{cccccc}
\multicolumn{7}{c}{ LCMV-infected targets } \\
$100: 1^{\mathrm{B}}$ & $50: 1$ & $25: 1$ & $12.5: 1$ & $5: 1$ & $1: 1$ \\
$67 \pm 2^{\mathrm{C}}$ & $57 \pm 9$ & $37 \pm 1$ & $14 \pm 7$ & NT & NT \\
$1 \pm 1$ & 1 & $1 \pm 1$ & 0 & NT & NT \\
NT & NT & NT & NT & 0 & 0
\end{tabular}

${ }^{A} A$ five-hour ${ }^{51} \mathrm{Cr}$ release assay was performed on $\mathrm{H}-2^{\mathrm{b}}$ uninfected target cells without $\mathrm{H}-2 \mathrm{D}^{\mathrm{b}}$ or coated with $\mathrm{H}-2 \mathrm{D}^{\mathrm{b}}$ restricted $\mathrm{LCMV}$ peptides $\mathrm{GP}$ aa $33-41$ and $\mathrm{NP}$ aa 396-404. Lymphocytes harvested from the spleen were added at effector cell to target cell ratios of 100:1, 50:1, 25:1, and 12.5:1. All assays were performed in triplicate with a variance less than $10 \%$. Displayed is the mean $\pm 1 \mathrm{SEM}$ of three or four mice in each experimental group. ${ }^{\mathrm{B}}$ Effector to target cell ratio. ${ }^{\mathrm{C}}$ Specific ${ }^{51} \mathrm{Cr}$ released from target cells after subtraction of release from allogeneic target cells $(11)$. ${ }^{\circ L C M V ~}(\mathrm{moi}=1)$ was used to infect syngeneic $\mathrm{H}-2^{\mathrm{b}}$ or allogeneic $\mathrm{H}-2^{\mathrm{d}}$ target cells also labeled with ${ }^{51} \mathrm{Cr}$. Effector cell to target cell ratios, use of triplicate samples, and calculation of specific ${ }^{51} \mathrm{Cr}$ release is as described in Methods and our publications $(11,19,22)$. NT, not tested.

The infiltration of $\mathrm{T}$ cells into the islets and subsequent islet destruction paralleled the occurrence of IDDM in RIP-IL12 $\times$ RIP-LCMV double-tg mice. In RIP-IL12 $\times$ RIP-GP mice, $\mathrm{CD} 8^{+}$and $\mathrm{CD} 4^{+} \mathrm{T}$ cells infiltrated the islets over a 12-day period after LCMV challenge (Figure 6). In RIP-IL12 $\times$ RIP-NP double-tg mice, $\mathrm{T}$ cell infiltration into islets occurred at a later timepoint (not shown). In conjunction with enhanced kinetics of disease incidence and absence of pancreatic insulin staining, a more severe infiltration of $\mathrm{CD}^{+}$and $\mathrm{CD}^{+} \mathrm{T}$ cells was observed in RIP-IL12 $\times$ RIP-NP tg mice than in RIP-NP single-tg littermates.

\section{Discussion}

In this paper, we report three novel findings. First, tg mice have been established in which the cytokine IL-12, in its bioactive form, is ectopically expressed and secreted in $\beta$ cells of the islets of Langerhans. IL- 12 is a pivotal regulator of both the innate and the adoptive immune responses (1). Second, in the RIP-LCMV model of $\operatorname{IDDM}(22,24), \mathrm{IL}-12$ produced in $\beta$ cells is unable to break tolerance to potentially autoreactive anti-self/viral $\mathrm{T}$ cells in the periphery, or to cause biochemical evident injury to the endocrine and exocrine pancreas (i.e., elevated levels of blood glucose, lipase, or amylase). However, marked infiltration of mononuclear cells into the pancreas, primarily to peri-islet locations, was associated with ectopic IL-12 expression. Third, IL-12 made in $\beta$ cells significantly accelerates the progression and magnifies the intensity of previously initiated IDDM.

Biochemical and molecular genetic analysis has documented that pancreatic islets of RIP-IL12 tg mice expressed and secreted functional IL-12 composed of the covalently linked subunits p35 and p40 (2). One consequence of focal expression of IL-12 in $\beta$ cells was the recruitment of $T$ and $B$ lymphocytes and antigen-presenting cells into the pancreas, predominantly localized around islets. The major inflammatory cells were $\mathrm{CD}^{+}$ $\mathrm{T}$ cells, which outnumbered $\mathrm{CD} 8^{+} \mathrm{T}$ cells by fivefold. Interestingly, both $\mathrm{CD}^{+}$and $\mathrm{CD} 8^{+} \mathrm{T}$ cells were inhibited from entering the islets where IL- 12 was being made, resembling the early prediabetic picture in NOD mice (25) and in tg mice that express LT- $\alpha$ (26) or IL-2 (27) in pancreatic $\beta$ cells. Hence, IL-12 alone is not a sufficient signal to allow trafficking of mononuclear cells into the islets of Langerhans. Macrophages were also found in abundance in the pancreatic infiltrates of RIP-IL12 tg mice. Their recruitment occurred independently of the presence of lymphocytes, since they were observed in the pancreata of RIP-IL12 $\times$ RAG-1-- mice. In tg mice that expressed only p 35 or p 40 , infiltration of mononuclear cells into the pancreas was not observed.

Trafficking of mononuclear cells into the pancreata of RIP-IL12 tg mice was associated with a marked upregulation of chemokine transcripts, especially RANTES, which was enhanced more than 60-fold compared with levels in non-tg littermates. The CCR5 receptor for RANTES (28) is preferentially expressed by Th1 lymphocytes (29-31). Additional ligands for CCR5 are MIP- $1 \alpha$ and MIP- $1 \beta$, both of which were upregulated in the pancreata of RIP-IL12 tg mice. Furthermore, IP-10 gene expression was enhanced 16-fold over that of control mice. IP-10 is a ligand for the CXCR3 chemokine receptor, which has been reported to be a 

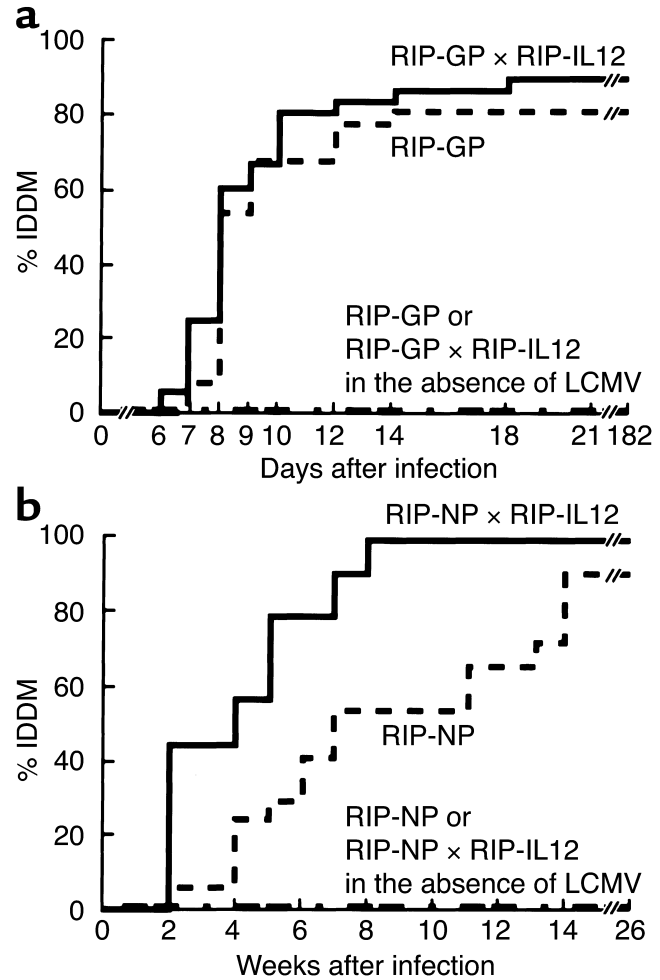

specific marker for Th1 lymphocytes $(30,31)$. In RIP-IL12 tg mice, it is likely that IP-10 was induced by the pancreatic expression of IFN- $\gamma(32)$.

Occurring with the accumulation of Th1 cells was the enhanced expression of IFN- $\gamma$, TNF- $\alpha$, LT- $\beta$, and IL- $1 \beta$ in the pancreas. Our finding of a concomitant 16 -fold enhanced production of IL-10 in RIP-IL12 tg mice extends an earlier discovery that IL-10 was induced by IL-12 in vivo (33). Besides IL-10, no other Th2 cytokine, i.e., IL-4, IL-5, IL-9, or IL-13, was induced by focal expression of IL-12 in vivo. These results complement our earlier findings in which IL10 expression in $\beta$ cells (RIP-IL10 tg mice) did not suppress virus-induced IDDM in RIP-LCMV mice (34), but IL4 expression in $\beta$ cells did (35). In contrast

\section{Figure 5}

Incidence of IDDM in RIP-LCMV and RIP-IL12 $\times$ RIP-LCMV tg mice after LCMV infection. Incidence of IDDM for RIP-IL12 $\times$ RIP-GP (a) and RIP-IL12 $\times$ RIP-NP (b) mice following infection with $10^{5} \mathrm{PFU}$ of LCMV, administered IP. In the absence of viral infection, none of 70 RIP-GP mice, 43 RIP-IL12 $\times$ RIP-GP mice, 25 RIP-NP mice, or 19 RIP-IL12 $\times$ RIP-NP mice developed elevated blood glucose levels, including some of these mice that were observed for more than 6 months ( $n=5$ for each group). After LCMV infection, IDDM developed in all RIP-LCMV lines, but with different kinetics and severity. In rapid-onset IDDM RIP-GP mice, in which the viral transgene is expressed only in $\beta$ cells of the islets of Langerhans, the incidence and kinetics of IDDM were roughly equivalent, although a quicker and more severe IDDM afflicted the double-tg RIP-IL12 × RIP-GP mice compared with single-tg RIP-GP mice. In the slow-onset IDDM RIP-NP mice, in which the viral transgene is expressed in the thymus as well as $\beta$ cells, IDDM arose more quickly and severely in the RIP-IL12 $\times$ RIP-NP mice than in single-tg RIP-NP littermates. Mice in each experimental group were age- and sex-matched, and were infected with LCMV at 6-8 weeks of age.

to the RIP-LCMV model (34), Balasa et al. (36) reported that focal expression of IL-10 in $\beta$ cells of NOD mice resulted in enhancement of diabetes, while in agreement with the RIP-LCMV model $(35,37), \beta$ cell expression of IL-4 protected NOD mice from autoimmune disease (38).

Unlike IFN- $\gamma$ (14) or B7.1 (15), IL-12 in $\beta$ cells failed to break the tolerance of antigen-specific $\mathrm{CD}^{+} \mathrm{T}$ cells to LCMV-("self")-antigens in RIP-LCMV tg mice. However, like these molecules and IL-2 (39), once autoimmune disease was initiated with LCMV infection, the activation of antigen-specific LCMV T cells accelerated, followed by quickened kinetics and severity of IDDM that was greater than that in single-tg RIP-LCMV mice. This observation was dramatic for slow-onset IDDM in which the prolonged developmental time facilitated analysis of IL-12's effect. Our results showing that the Th1-promoting cytokine IL-12 accelerates autoimmune diabetes complement earlier studies in which exogenous administration of IL-12 accelerated autoimmune diabetes in NOD mice

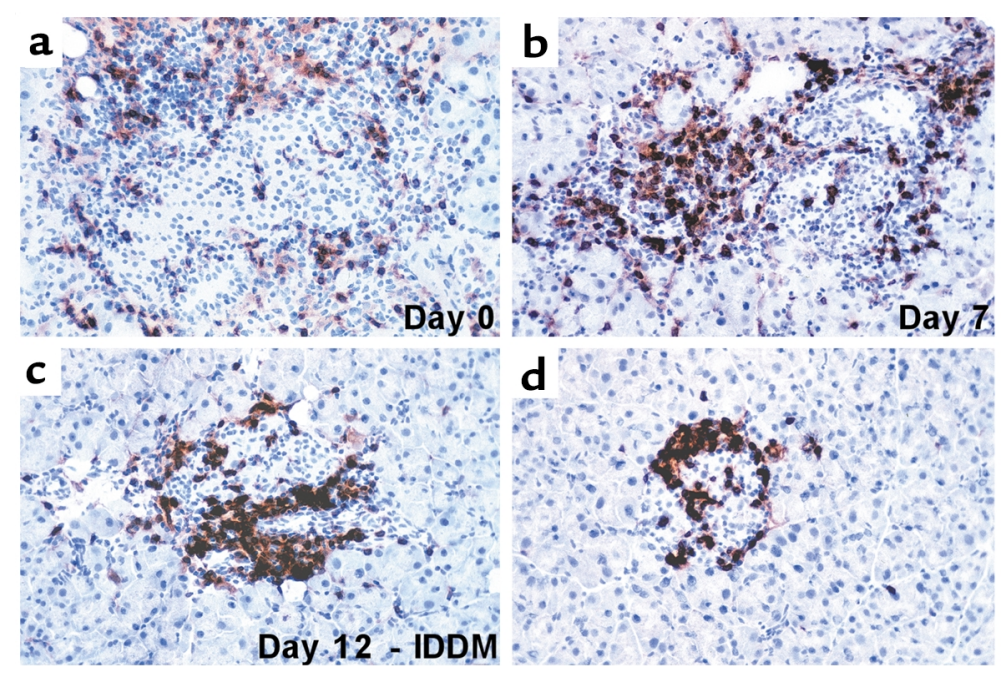

\section{Figure 6}

Infiltration of T lymphocytes into the pancreata of RIP-IL12 × RIP-GP double-tg mice. Photomicrographs of immunohistochemical localization of $\mathrm{CD}^{+} \mathrm{T}$ cells into the islets of RIP-IL $12 \times$ RIP-GP mice (a-c) and into a single-tg RIP-GP littermate (d). The RIP-IL12 $\times$ RIP-GP mouse whose pancreas is shown in a was not infected with LCMV, and displays $C D 8^{+}$ $T$ cells in peri-islet locations. In contrast, the islets shown in $\mathbf{b}-\mathbf{d}$ were obtained from mice 7 days (b) and 12 days ( $\mathbf{c}$ and $\mathbf{d}$ ) after infection with LCMV, and show $\mathrm{CD} 8^{+} \mathrm{T}$ cells infiltrating into islets. Findings were similar in adjacent tissue sections stained for $\mathrm{CD}^{+} \mathrm{T}$ lymphocytes. Results are representative of four mice from each group. 
(12). Although Th1-promoting cytokines mainly accelerate autoimmune processes, they may also limit autoimmune destruction (40).

Understanding how immunologic tolerance is broken or restored is important for treating autoimmune diseases (41). RIP-LCMV tg mice are an excellent model for this purpose $(22,42)$, because the viral transgene serves as a marker of self-antigen, the effector $\mathrm{T}$ cell responses to which can be dissected in exquisite detail (43). This tg model, with bioactive IL-12 secreted by pancreatic $\beta$ cells, offered the opportunity to evaluate whether IL-12 itself was sufficient to break tolerance/unresponsiveness and initiate autoimmune disease. Our results indicate that ectopic expression of IL-12 in $\beta$ cells did not spontaneously activate anti-self/viral T cells, since no anti-LCMV CTL response developed, and no autoimmune diabetes occurred. We previously noted that one or more antigen-specific cells per 5,000 T cells was required to cause IDDM (44). When B7.1 was expressed in $\beta$ cells in RIP-B7.1 tg mice (45), no islet injury occurred, just as in the RIP-IL12 mice reported here. However, unlike RIP-IL12 $\times$ RIP-GP double-tg mice, RIP-B7.1 $\times$ RIP-GP mice spontaneously activated antigen-specific anti-self/viral $\mathrm{CD}^{+} \mathrm{T}$ cells to a number of 1 per 3,000 total $\mathrm{CD}^{+} \mathrm{T}$ cells, which was sufficient to cause spontaneous IDDM (15). In addition to B7.1, expression of IFN- $\gamma$ in $\beta$ cells also broke unresponsiveness to antigen-specific (anti-LCMV) $\mathrm{CD}^{+} \mathrm{T}$ cells in the RIP-LCMV tg mice (14). However, in contrast to RIP-B7.1 mice, in which there is no evidence of islet destruction, tissue injury of islets occurred in RIP-IFN- $\gamma$ mice (16), and spontaneously activated anti-islet $\mathrm{T}$ cells were found (17). This process was exacerbated in RIP-IFN- $\gamma \times$ RIP-LCMV tg mice, in which antigen-specific anti-LCMV CD8 ${ }^{+} \mathrm{T}$ cells were also generated spontaneously (14). Apparently, either the amount of IFN- $\gamma$ induced by IL- 12 expression in the RIP-IL-12 mice was not sufficient to break peripheral unresponsiveness, or tissue injury in the islets of RIP-IFN- $\gamma$ mice was caused by expression of the transgene in $\beta$ cells, possibly involving upregulation of costimulatory molecules, epitope spreading (46), and activation of anti-islet $\mathrm{T}$ cells that caused spontaneous IDDM. The inability of IL-12 to break peripheral tolerance/unresponsiveness is similar to that of IL-2 (27) or TNF- $\alpha$ (47) expressed in $\beta$ cells.

Our study suggests that in addition to IL-12 expression in $\beta$ cells, other signals are required to break unresponsiveness to self-antigens in vivo. In contrast to $\operatorname{IFN}-\gamma(16,17,48)$, focal expression of IL-12 might be unable to upregulate sufficient amounts of costimulatory molecules to induce diabetes. When B7.1 and IL- 12 were coexpressed in $\beta$ cells of tg mice, diabetes occurred in $100 \%$ of RIP-IL12 $\times$ RIP-B7.1 double-tg offspring at 6-8 weeks of age (A. Holz and M.B.A. Oldstone, unpublished observations). In contrast, single-tg littermates of RIP-IL12 or RIP-B7.1 mice did not develop diabetes.
The lack of $\beta$ cell or islet injury and disease in RIP-IL12 tg mice contrasts with a recent report (49) that IL-12 expressed in astrocytes of the CNS under transcriptional control of the glial fibrillary acidic protein (GFAP) promoter caused neurologic disease and reduced the life span of the animals. Still unclear is whether disease in GFAP-IL-12 tg mice resulted from the toxicity of transgene expression to astrocytes or activation of anti-self CNS T cells.

In tg mice with IL-12 focally expressed in the islets, one can identify additional signals needed for $T$ cells to enter the islets. Our unpublished work indicates that this deficiency is not from lack of expression of adhesion molecules, e.g., ICAM-1 (Holz and Oldstone, unpublished observations). In addition, the availability of both MHC class I and class II tetramers to LCMV antigens (50) (Teyton and Oldstone, unpublished observations), coupled with gene display assays like TOGA (51) or other gene analysis techniques, promises to provide a clearer molecular definition and potential for effective treatment of IDDM.

\section{Acknowledgments}

We thank Diane Thomas for excellent technical assistance, and Nora Sarvetnick for helpful discussions. This work was supported by NIH public health grant AI-41439. A. Holz was supported consecutively by Swiss National Foundation advanced fellowship 823A050437, and by National Multiple Sclerosis Society advanced research fellowship award FA1333-A1.

1. Trinchieri, G. 1998. Interleukin-12: a cytokine at the interface of inflammation and immunity. Adv. Immunol. 70:83-243.

2. Kobayashi, M., et al. 1989. Identification and purification of natural killer cell stimulatory factor (NKSF), a cytokine with multiple biologic effects on human lymphocytes. J. Exp. Med. 170:827-845.

3. Chan, S.H., et al. 1991. Induction of interferon gamma production by natural killer cell stimulatory factor: characterization of the responder cells and synergy with other inducers. J. Exp. Med. 173:869-879.

4. Manetti, R., et al. 1993. Natural killer cell stimulatory factor (interleukin 12 [IL-12]) induces T helper type 1 (Th1)-specific immune responses and inhibits the development of IL-4-producing Th cells. J. Exp. Med. 177:1199-1204.

5. Hsieh, C.S., et al. 1993. Development of TH1 CD4+ T cells through IL12 produced by Listeria-induced macrophages. Science. 260:547-549.

6. Afonso, L.C., et al. 1994. The adjuvant effect of interleukin-12 in a vaccine against Leishmania major. Science. 263:235-237.

7. Adorini, L. 1999. Interleukin-12, a key cytokine in Th1-mediated autoimmune diseases. Cell. Mol. Life Sci. 55:1610-1625.

8. Caspi, R.R. 1998. IL-12 in autoimmunity. Clin. Immunol. Immunopathol. 88:4-13.

9. Leonard, J.P., Waldburger, K.E., and Goldman, S.J. 1995. Prevention of experimental autoimmune encephalomyelitis by antibodies against interleukin 12. J. Exp. Med. 181:381-386.

10. Segal, B.M., Dwyer, B.K., and Shevach, E.M. 1998. An interleukin (IL)10/IL-12 immunoregulatory circuit controls susceptibility to autoimmune disease. J. Exp. Med. 187:537-546.

11. Holz, A., et al. 1999. Disruption of the STAT4 signaling pathway protects from autoimmune diabetes while retaining antiviral immune competence. J. Immunol. 163:5374-5382.

12. Trembleau, S., et al. 1995. Interleukin 12 administration induces $T$ helper type 1 cells and accelerates autoimmune diabetes in NOD mice. J. Exp. Med. 181:817-821.

13. Smith, T., Hewson, A.K., Kingsley, C.I., Leonard, J.P., and Cuzner, M.L. 1997. Interleukin-12 induces relapse in experimental allergic encephalomyelitis in the Lewis rat. Am.J. Pathol. 150:1909-1917.

14. Lee, M.S., von Herrath, M.G., Reiser, H., Oldstone, M.B., and Sarvetnick, N. 1995. Sensitization to self (virus) antigen by in situ expression of murine interferon- $\gamma$. J. Clin. Invest. 95:486-492.

15. von Herrath, M.G., Guerder, S., Lewicki, H., Flavell, R.A., and Oldstone, 
M.B. 1995. Coexpression of B7-1 and viral ("self") transgenes in pancreatic beta cells can break peripheral ignorance and lead to spontaneous autoimmune diabetes. Immunity. 3:727-738.

16. Sarvetnick, N., Liggitt, D., Pitts, S.L., Hansen, S.E., and Stewart, T.A. 1988. Insulin-dependent diabetes mellitus induced in transgenic mice by ectopic expression of class II MHC and interferon-gamma. Cell. 52:773-782.

17. Sarvetnick, N., et al. 1990. Loss of pancreatic islet tolerance induced by beta-cell expression of interferon-gamma. Nature. 346:844-847.

18. Mombaerts, P., et al. 1992. RAG-1-deficient mice have no mature B and T lymphocytes. Cell. 68:869-877.

19. von Herrath, M.G., Dockter, J., and Oldstone, M.B. 1994. How virus induces a rapid or slow onset insulin-dependent diabetes mellitus in a transgenic model. Immunity. 1:231-242.

20. Holz, A., et al. 2000. Neither B-lymphocytes nor antibodies directed against self antigens of the islets of Langerhans are required for development of virus-induced autoimmune diabetes. J. Immunol. 165:5945-5953.

21. Seewaldt, S., et al. 2000. Virus-induced autoimmune diabetes: most betacells die through inflammatory cytokines and not perforin from autoreactive (anti-viral) cytotoxic T-lymphocytes. Diabetes. 49:1801-1809.

22. Oldstone, M.B., Nerenberg, M., Southern, P., Price, J., and Lewicki, H. 1991. Virus infection triggers insulin-dependent diabetes mellitus in a transgenic model: role of anti-self (virus) immune response. Cell. 65:319-331.

23. von Herrath, M.G., Dockter, J., Nerenberg, M., Gairin, J.E., and Oldstone, M.B. 1994. Thymic selection and adaptability of cytotoxic T lymphocyte responses in transgenic mice expressing a viral protein in the thymus. $J$. Exp. Med. 180:1901-1910.

24. Ohashi, P.S., et al. 1991. Ablation of "tolerance" and induction of diabetes by virus infection in viral antigen transgenic mice. Cell. 65:305-317.

25. Bach, J.F. 1994. Insulin-dependent diabetes mellitus as an autoimmune disease. Endocr. Rev. 15:516-542.

26. Picarella, D.E., Kratz, A., Li, C.B., Ruddle, N.H., and Flavell, R.A. 1992. Insulitis in transgenic mice expressing tumor necrosis factor beta (lymphotoxin) in the pancreas. Proc. Natl. Acad. Sci. USA. 89:10036-10040.

27. Allison, J., Malcolm, L., Chosich, N., and Miller, J.F. 1992. Inflammation but not autoimmunity occurs in transgenic mice expressing constitutive levels of interleukin-2 in islet beta cells. Eur. J. Immunol. 22:1115-1121.

28. Meyer, A., Coyle, A.J., Proudfoot, A.E., Wells, T.N., and Power, C.A. 1996. Cloning and characterization of a novel murine macrophage inflammatory protein-1 alpha receptor. J. Biol. Chem. 271:14445-14451.

29. Bonecchi, R., et al. 1998. Differential expression of chemokine receptors and chemotactic responsiveness of type $1 \mathrm{~T}$ helper cells (Th1s) and Th2s. J. Exp. Med. 187:129-134.

30. Qin, S., et al. 1998. The chemokine receptors CXCR3 and CCR5 mark subsets of T cells associated with certain inflammatory reactions. J. Clin. Invest. 101:746-754.

31. Sallusto, F., Lenig, D., Mackay, C.R., and Lanzavecchia, A. 1998. Flexible programs of chemokine receptor expression on human polarized $\mathrm{T}$ helper 1 and 2 lymphocytes. J. Exp. Med. 187:875-883.

32. Luster, A.D., and Ravetch, J.V. 1987. Biochemical characterization of a gamma interferon-inducible cytokine (IP-10). J. Exp. Med. 166:1084-1097.

33. Finkelman, F.D., et al. 1994. Effects of interleukin 12 on immune responses and host protection in mice infected with intestinal nematode parasites. J. Exp. Med. 179:1563-1572.
34. Lee, M.S., Wogensen, L., Shizuru, J., Oldstone, M.B., and Sarvetnick, N. 1994. Pancreatic islet production of murine interleukin-10 does not inhibit immune-mediated tissue destruction. J. Clin. Invest. 93:1332-1338.

35. von Herrath, M.G., and Oldstone, M.B.A. 1996. Virus-induced autoimmune disease. Curr. Opin. Immunol. 8:878-885.

36. Balasa, B., et al. 2000. Islet-specific expression of IL-10 promotes diabetes in nonobese diabetic mice independent of Fas, perforin, TNF receptor1 , and receptor-2 molecules. J. Immunol. 165:2841-2849.

37. King, C., et al. 2001. Interleukin- 4 acts at the locus of the antigen-presenting dendritic cell to counter-regulate cytotoxic CD8+ T-cell responses. Nat. Med. 7:206-214.

38. Mueller, R., Krahl, T., and Sarvetnick, N. 1996. Pancreatic expression of interleukin-4 abrogates insulitis and autoimmune diabetes in nonobese diabetic (NOD) mice. J. Exp. Med. 184:1093-1099.

39. von Herrath, M.G., Allison, J., Miller, J.F., and Oldstone, M.B. 1995. Focal expression of interleukin-2 does not break unresponsiveness to "self" (viral) antigen expressed in beta cells but enhances development of autoimmune disease (diabetes) after initiation of an anti-self immune response. J. Clin. Invest. 95:477-485.

40. Falcone, M., and Sarvetnick, N. 1999. Cytokines that regulate autoimmune responses. Curr. Opin. Immunol. 11:670-676.

41. Bach, J.F., and Chatenoud, L. 2001. Tolerance to islet autoantigens in type 1 diabetes. Annu. Rev. Immunol. 19:131-161.

42. von Herrath, M.G., Homann, D., Gairin, J.E., and Oldstone, M.B.A. 1997. Pathogenesis and treatment of virus-induced autoimmune diabetes: novel insights gained from the RIP-LCMV transgenic mouse model Biochem. Soc. Trans. 25:630-635.

43. Borrow, P., and Oldstone, M.B.A. 1997. Lymphocytic choriomeningitis virus. In Viral Pathogenesis. N. Nathanson et al., editors. Lippincott-Raven Publishers. Philadelphia, Pennsylvania, USA. 593-627.

44. Sevilla, N., et al. 2000. Virus-induced diabetes in a transgenic model: role of cross-reacting viruses and quantification of effector $\mathrm{T}$ cells needed to cause disease. J. Virol. 74:3284-3292.

45. Guerder, S., Meyerhoff, J., and Flavell, R. 1994. The role of the T cell costimulator B7-1 in autoimmunity and the induction and maintenance of tolerance to peripheral antigen. Immunity. 1:155-166.

46. McRae, B.L., Vanderlugt, C.L., Dal Canto, M.C., and Miller, S.D. 1995 Functional evidence for epitope spreading in the relapsing pathology of experimental autoimmune encephalomyelitis. J. Exp. Med. 182:75-85.

47. Picarella, D.E., Kratz, A., Li, C.B., Ruddle, N.H., and Flavell, R.A. 1993 Transgenic tumor necrosis factor (TNF)-alpha production in pancreatic islets leads to insulitis, not diabetes. Distinct patterns of inflammation in TNF-alpha and TNF-beta transgenic mice. J. Immunol. 150:4136-4150.

48. von Herrath, M.G., and Oldstone, M.B.A. 1997. Interferon- $\gamma$ is essential for destruction of $\beta$-cells and development of insulin-dependent diabetes mellitus. J. Exp. Med. 185:531-539.

49. Pagenstecher, A., et al. 2000. Astrocyte-targeted expression of IL-12 induces active cellular immune responses in the central nervous system and modulates experimental allergic encephalomyelitis. J. Immunol. 164:4481-4492.

50. Homann, D., Teyton, L., and Oldstone, M.B.A. 2001. Differential regulation of antiviral T-cell immunity results in stable CD8+ but declining CD4+ T-cell memory. Nat. Med. 7:913-919.

51. Sutcliffe, J.G., et al. 2000. TOGA: an automated parsing technology for analyzing expression of nearly all genes. Proc. Natl. Acad. Sci. USA 97:1976-1981. 